Editorial

\title{
New Journal Launch: Global Clinical and Translational Research
}

Accepted October 30, 2018

We are proud to announce the launch of Global Clinical and Translational Research, a new journal that aims to promote clinical and translational research in the genomic era from a global perspective. The journal will (1) provide a unified platform of research communication for basic scientists, medical doctors, other clinical health professionals, social scientists and social workers to share the most recent advances in all areas of clinical and translational sciences; (2) introduce new techniques and methodology such as genomics-based techniques and approaches to design and perform a new generation of clinical research; (3) foster collaborative clinical research from a global perspective.

In the first two decades of the $21^{\text {st }}$ century, strategies for conducting biomedical research have been profoundly influenced by the completion of the Human Genome Projects (HGP) [1]. The HGP is properly regarded as the major groundbreaking accomplishment in human biology as we moved from the $20^{\text {th }}$ into the $21^{\text {st }}$ century. Related projects such as the International Haplotype Map of the Human Genome Project (HapMap) and the development of the tools of bioinformatics computer programs, especially the invention of genome-wide association techniques, have made it possible to dissect genetic architecture of complex human diseases and health, and to identify genetic variants that may impact the response of individuals or populations to pharmaceutical treatments, environmental exposures to chemicals or toxicants and psychosocial stressors. The findings from such research over the past decade have accelerated discoveries such as identification of molecular targets of drugs and other environmental or dietary compounds as well as novel targets for further research and development of other ligands that may become new therapeutic medications including and offer new evidence for population-based interventions, or early and precision diagnostics.

With the increasingly available tools of bio-technology in genomics and other "omics," the then U.S. President Obama announced the precision medicine initiative in 2015. The goal of this initiative is to pioneer a new model of biomedical and health research that promises to acelerate biomedical discoveries and translate them into new tools, knowledge, and therapies for clinicians to select which treatments will work best for which patients and for public health professionals to better decide when and how preventative measures should be taken. The concept of precision medicine is to develop treatment and prevention strategies that take individual variability [2] into account, which may involve the individual's genetic heterogeneity, life course experiences, and lifestyle. Identifying those individual factors will require a series of research activities across different populations to accomm- odate variation in the genetic background including ethnicity, as well as local or regional environmental and dietary factors.

This new journal will be a vehicle to communicate those new findings of clinical and translational research in the era of genomics or other "omics." Without a doubt, following the precision medicine initiative, clinical and translational research will be a robust global enterprise over the next few decades; therefore, with wide application of the new tools of biotechnology, more and more studies will focus on discovery in human subjects through observational and experimental approaches. Findings from such studies can be translated not only into basic research hypotheses that should lead to novel ideas for mechanistic biological research or biology but also into clinical practices such as precision diagnosis and treatment, preventive interventions at a population level or enhanced environmental health for entire communities [3]. The scope of translational research must greatly extend beyond the traditionally defined "from bench to bedside and back again" to a broad multidisciplinary and multilayered process of discovery, implementation, and global public health impact.

The journal will be edited by a multidisciplinary team lead by Drs. Claude Hughes and Fengyu Zhang. Dr. Hughes' broad experience includes pharmaceutical clinical trials; practice of Obstetrics \& Gynecology and Reproductive Endocrinology \& Infertility; modeling and biomarker development; basic, clinical and population research in the health of women across the lifespan, and the impact of dietary and environmental chemicals in reproductive, developmental and translational toxicology. Dr. Zhang's research expertise focus conducting multidisciplinary research on genetic and environmental determinants of complex human disorders and population health, clinical trial-based pharmacogenomics of treatment response, as well as biomarker discovery; and he has a strong background in quantitative and data science.

The journal accepts original research articles, reviews, mini-reviews, case reports, short notes, and rapid communications covering all aspects of clinical and translational research. Papers about novel applications of statististical methods or data science are also welcomed.

Editors-in-Chief Claude Hughes, MD PhD Fengyu Zhang, PhD MS

\section{REFERENCES}


1. Collins FS, Morgan M, Patrinos A. The Human Genome Project: lessons from large-scale biology. Science. 2003; 300 (5617):286-90.

2. Collins FS, Varmus H. A new initiative on precision medicine. N Engl J Med. 2015;372(9):793-5.
3. Collman GW, Berridge BR, Hall JE, Woychik R, Zeldin DC, Birnbaum LS. NIEHS: making a mark on translational research science. Environ Health Perspect. 2018;126 (8): 081001.

How to cite the article:

Hughes C, Zhang F. New Journal Launch: Global Clinical and Translational Research. Glob Clin Transl Res. 2019; 1(1):2-3.

Copyright (C) 2019 by the Global Clinical and Translational Research. 